PROCEEDINGS OF THE

AMERICAN MATHEMATICAL SOCIETY

Volume 128, Number 7, Pages 2085-2094

S 0002-9939(00)05320-X

Article electronically published on February 25, 2000

\title{
TWIN SOLUTIONS \\ TO SINGULAR BOUNDARY VALUE PROBLEMS
}

\author{
RAVI P. AGARWAL AND DONAL O'REGAN
}

(Communicated by Hal L. Smith)

\begin{abstract}
In this paper we establish the existence of two nonnegative solutions to singular $(n, p)$ and singular $(p, n-p)$ focal boundary value problems. Our nonlinearity $f(t, y)$ may be singular at $y=0, t=0$ and/or $t=1$.
\end{abstract}

\section{INTRODUCTION}

This paper discusses the existence of multiple nonnegative solutions to the singular $(n, p)$ boundary value problem

$$
\left\{\begin{array}{l}
y^{(n)}(t)+\phi(t) f(t, y(t))=0,0<t<1, \\
y^{(i)}(0)=0, \quad 0 \leq i \leq n-2, \\
y^{(p)}(1)=0
\end{array}\right.
$$

and the singular $(p, n-p)$ focal boundary value problem

$$
\left\{\begin{array}{l}
(-1)^{n-p} y^{(n)}(t)=\phi(t) f(t, y(t)), \quad 0<t<1 \\
y^{(i)}(0)=0, \quad 0 \leq i \leq p-1 \\
y^{(i)}(1)=0, \quad p \leq i \leq n-1
\end{array}\right.
$$

here $n \geq 2$ and $1 \leq p \leq n-1$ is fixed. All the papers in the literature on singular problems (see [1]-[7] and their references) are devoted to establishing the existence of one solution to singular boundary value problems. This is the first paper, to our knowledge, that establishes the existence of more than one solution to (1.1) and (1.2) even in the case when $n=2$. The technique presented to guarantee the existence of twin nonnegative solutions to (1.1) and (1.2) is new and involves combining (i) an existence result from the literature (which relies on a Leray-Schauder alternative), (ii) Krasnoselskii's fixed point theorem in a cone, and (iii) lower type inequalities.

For the remainder of this introduction we present some results from the literature which will be needed in Section 2. Let $k(t, s)$ be the Green's function for

$$
\left\{\begin{array}{l}
-y^{(n)}=0 \quad \text { on }[0,1] \\
y^{(i)}(0)=0, \quad 0 \leq i \leq n-2, \\
y^{(p)}(1)=0
\end{array}\right.
$$

Received by the editors September 1, 1998.

1991 Mathematics Subject Classification. Primary 34B15.

Key words and phrases. Multiple solutions, singular problems, Leray-Schauder alternative, Krasnoselskii's fixed point theorem, lower type inequalities. 
see [4] for an explicit representation. It is well known [4] that

$$
k^{(i)}(t, s) \geq 0 \text { for }(t, s) \in[0,1] \times[0,1] \text { and } 0 \leq i \leq p ; \text { here } k^{(i)}=\frac{\partial^{i}}{\partial t^{i}} k .
$$

In $[3$ we proved the following lower type inequality.

Theorem 1.1. Suppose $y \in C^{n-1}[0,1] \cap C^{n}(0,1)$ satisfies

$$
\left\{\begin{array}{l}
y^{(n)}(t) \leq 0 \text { for } t \in[0,1] \\
y(0)=a \geq 0 \\
y^{(i)}(0)=0,1 \leq i \leq n-2, \\
y^{(p)}(1)=0
\end{array}\right.
$$

Then

$$
y(t) \geq t^{n-1}|y|_{0}\left(=t^{n-1} y(1)\right) \text { for } t \in[0,1] ;
$$

here $|y|_{0}=\sup _{t \in[0,1]}|y(t)|$.

Next let $G(t, s)$ be the Green's function for

$$
\left\{\begin{array}{l}
y^{(n)}=0 \text { on }[0,1], \\
y^{(i)}(0)=0, \quad 0 \leq i \leq p-1, \\
y^{(i)}(1)=0, \quad p \leq i \leq n-1
\end{array}\right.
$$

see 4 for an explicit representation. It is well known [4] that for $(t, s) \in[0,1] \times[0,1]$,

$$
(-1)^{n-p} G^{(i)}(t, s) \geq 0,0 \leq i \leq p-1,
$$

and

$$
(-1)^{n-i} G^{(i)}(t, s) \geq 0, p \leq i \leq n-1 .
$$

In 2] we proved the following lower type inequality.

Theorem 1.2. Suppose $y \in C^{n-1}[0,1] \cap C^{n}(0,1)$ satisfies

$$
\left\{\begin{array}{l}
(-1)^{n-p} y^{(n)}(t) \geq 0 \quad \text { for } t \in(0,1) \\
y(0)=a \geq 0 \\
y^{(i)}(0)=0,1 \leq i \leq p-1 \\
y^{(i)}(1)=0, p \leq i \leq n-1
\end{array}\right.
$$

Then

$$
y(t) \geq t^{p}|y|_{0}\left(=t^{p} y(1)\right) \quad \text { for } t \in[0,1] .
$$

In 2] we proved the following existence result for the singular $(p, n-p)$ focal problem (1.2).

Theorem 1.3. Suppose the following conditions are satisfied:

$$
\begin{aligned}
& \phi \in C(0,1) \text { with } \phi>0 \text { on }(0,1) \text { and } \phi \in L^{1}[0,1], \\
& f:[0,1] \times(0, \infty) \rightarrow(0, \infty) \text { is continuous, } \\
& \begin{cases}f(t, y) \leq g(y)+h(y) & \text { on }[0,1] \times(0, \infty) \text { with } g>0 \text { continuous } \\
\text { and nonincreasing on }(0, \infty), h \geq 0 \text { continuous } & \text { on }[0, \infty) \text { and } \frac{h}{g} \text { nondecreasing on }(0, \infty),\end{cases}
\end{aligned}
$$




$$
\left\{\begin{array}{l}
\text { for each constant } H>0 \text { there exists } \psi_{H} \text { continuous on }[0,1] \\
\text { and positive on }(0,1) \text { such that } f(t, y) \geq \psi_{H}(t) \text { on }[0,1] \times(0, H]
\end{array}\right.
$$

$$
\int_{0}^{1} \phi(s) g\left(s^{p}\right) d s<\infty
$$

there exists a constant $K_{0}>0$ with $g(a b) \leq K_{0} g(a) g(b)$ for all $a \geq 0, b \geq 0$, and

$$
\text { there exists a constant } r>0 \text { with } \frac{r}{g(r)+h(r)}>b_{0} K_{0} \text {; }
$$

here

$$
b_{0}=\sup _{t \in[0,1]} \int_{0}^{1}(-1)^{n-p} G(t, s) \phi(s) g\left(s^{p}\right) d s .
$$

Then (1.2) has a solution $y \in C^{n-1}[0,1] \cap C^{n}(0,1)$ with $y>0$ on $(0,1]$ and $|y|_{0}<r$.

Essentially the same reasoning as in [2] establishes the corresponding result for the singular $(n, p)$ problem (1.1).

Theorem 1.4. Suppose (1.7)-(1.10) and (1.12) hold. In addition assume

$$
\int_{0}^{1} \phi(s) g\left(s^{n-1}\right) d s<\infty
$$

and

$$
\text { there exists a constant } r>0 \text { with } \frac{r}{g(r)+h(r)}>c_{0} K_{0}
$$

are satisfied; here

$$
c_{0}=\sup _{t \in[0,1]} \int_{0}^{1} k(t, s) \phi(s) g\left(s^{n-1}\right) d s .
$$

Then (1.1) has a solution $y \in C^{n-1}[0,1] \cap C^{n}(0,1)$ with $y>0$ on $(0,1]$ and $|y|_{0}<r$.

Finally we state for completeness Krasnoselskii's fixed point theorem in a cone.

Theorem 1.5. Let $E=(E,\|\|$.$) be a Banach space and let K \subset E$ be a cone in $E$. Assume $\Omega_{1}$ and $\Omega_{2}$ are open subsets of $E$ with $0 \in \Omega_{1}$ and $\overline{\Omega_{1}} \subset \Omega_{2}$ and let $\left.A: K \cap \overline{\Omega_{2}} \backslash \Omega_{1}\right) \rightarrow K$ be continuous and completely continuous. In addition suppose either

$$
\|A u\| \leq\|u\| \text { for } u \in K \cap \partial \Omega_{1} \text { and }\|A u\| \geq\|u\| \text { for } u \in K \cap \partial \Omega_{2}
$$

or

$$
\|A u\| \geq\|u\| \text { for } u \in K \cap \partial \Omega_{1} \text { and }\|A u\| \leq\|u\| \text { for } u \in K \cap \partial \Omega_{2}
$$

hold. Then $A$ has a fixed point in $K \cap\left(\overline{\Omega_{2}} \backslash \Omega_{1}\right)$. 


\section{Singular PRoblems}

In this section we begin by discussing the singular $(n, p)$ problem

$$
\left\{\begin{array}{l}
y^{(n)}(t)+\phi(t)[g(y(t))+h(y(t))]=0,0<t<1, \\
y^{(i)}(0)=0,0 \leq i \leq n-2, \\
y^{(p)}(1)=0
\end{array}\right.
$$

with $n \geq 2$ and $1 \leq p \leq n-1$ is fixed.

Theorem 2.1. Assume the following conditions are satisfied:

$$
\begin{gathered}
\phi \in C(0,1) \text { with } \phi>0 \text { on }(0,1) \text { and } \phi \in L^{1}[0,1], \\
g>0 \text { is continuous and nonincreasing on }(0, \infty),
\end{gathered}
$$

$$
h \geq 0 \text { is continuous on }[0, \infty) \text { with } \frac{h}{g} \text { nondecreasing on }(0, \infty),
$$

$$
\int_{0}^{1} \phi(s) g\left(s^{n-1}\right) d s<\infty
$$

there exists a constant $K_{0}>0$ with $g(a b) \leq K_{0} g(a) g(b)$ for all $a \geq 0, b \geq 0$

and

$$
\text { there exists a constant } r>0 \text { with } \frac{r}{g(r)+h(r)}>c_{0} K_{0} \text {; }
$$

here

$$
c_{0}=\sup _{t \in[0,1]} \int_{0}^{1} k(t, s) \phi(s) g\left(s^{n-1}\right) d s
$$

where $k(t, s)$ is the Green's function for (1.3). Then (2.1) has a solution $y \in$ $C^{n-1}[0,1] \cap C^{n}(0,1)$ with $y>0$ on $(0,1]$ and $|y|_{0}<r$.

Proof. The result follows from Theorem 1.4 with $f(t, u)=g(u)+h(u)$. Notice (1.10) is clearly satisfied with $\psi_{H}(t)=g(H)$.

Theorem 2.2. Assume (2.2)-(2.7) hold. In addition suppose

$$
\text { there exists } R>r \text { with } \frac{R g\left(\frac{R}{4^{n-1}}\right)}{g(R) g\left(\frac{R}{4^{n-1}}\right)+g(R) h\left(\frac{R}{4^{n-1}}\right)} \leq \int_{\frac{1}{4}}^{1} k(\sigma, s) \phi(s) d s \text {; }
$$

here $0 \leq \sigma \leq 1$ is such that

$$
\int_{\frac{1}{4}}^{1} k(\sigma, s) \phi(s) d s=\sup _{t \in[0,1]} \int_{\frac{1}{4}}^{1} k(t, s) \phi(s) d s .
$$

Then (2.1) has a solution $y \in C^{n-1}[0,1] \cap C^{n}(0,1)$ with $y>0$ on $(0,1]$ and $r<|y|_{0} \leq R$. 
Remark 2.1. It is possible to replace (2.9) with

there exists $R>r$ with $\frac{R g\left(R a^{n-1}\right)}{g(R) g\left(R a^{n-1}\right)+g(R) h\left(R a^{n-1}\right)} \leq \int_{a}^{1} k(\eta, s) \phi(s) d s ;$ here $0<a<\frac{1}{2}$ is fixed and $\eta \in[0,1]$ is such that

$$
\int_{a}^{1} k(\eta, s) \phi(s) d s=\sup _{t \in[0,1]} \int_{a}^{1} k(t, s) \phi(s) d s .
$$

Proof. To show the existence of the solution described in the statement of Theorem 2.2 we will apply Theorem 1.5. First however choose $\epsilon>0$ and $\epsilon<r$ with

$$
\frac{r}{\epsilon+c_{0} K_{0}[g(r)+h(r)]}>1 \text {. }
$$

Let $m_{0} \in\{1,2, \ldots\}$ be chosen so that $\frac{1}{m_{0}}<\epsilon$ and $\frac{1}{m_{0}}<\frac{R}{4^{n-1}}$ and let $N_{0}=$ $\left\{m_{0}, m_{0}+1, \ldots\right\}$. We first show that

$$
\left\{\begin{array}{l}
y^{(n)}(t)+\phi(t)[g(y(t))+h(y(t))]=0,0<t<1, \\
y(0)=\frac{1}{m} \\
y^{(i)}(0)=0, \quad 1 \leq i \leq n-2, \\
y^{(p)}(1)=0
\end{array}\right.
$$

has a solution $y_{m}$ for each $m \in N_{0}$ with $y_{m}>\frac{1}{m}$ on $(0,1]$ and $r \leq\left|y_{m}\right|_{0} \leq R$.

To show $(2.12)^{m}$ has such a solution for each $m \in N_{0}$, we will look at

$$
\left\{\begin{array}{l}
y^{(n)}(t)+\phi(t)\left[g^{\star}(y(t))+h(y(t))\right]=0,0<t<1 \\
y(0)=\frac{1}{m} \\
y^{(i)}(0)=0, \quad 1 \leq i \leq n-2 \\
y^{(p)}(1)=0
\end{array}\right.
$$

with

$$
g^{\star}(u)=\left\{\begin{array}{l}
g(u), \quad u \geq \frac{1}{m}, \\
g\left(\frac{1}{m}\right), \quad 0 \leq u \leq \frac{1}{m} .
\end{array}\right.
$$

Remark 2.2. Notice $g^{\star}(u) \leq g(u)$ for $u>0$.

Fix $m \in N_{0}$. Let $E=\left(C[0,1],|\cdot|_{0}\right)$ and

$$
K=\left\{u \in C[0,1]: u(t) \geq 0 \text { for } t \in[0,1] \text { and } u(t) \geq t^{n-1}|u|_{0} \text { for } t \in[0,1]\right\} .
$$

Clearly $K$ is a cone of $E$. Let $A: K \rightarrow C[0,1]$ be defined by

$$
A y(t)=\frac{1}{m}+\int_{0}^{1} k(t, s) \phi(s)\left[g^{\star}(y(s))+h(y(s))\right] d s .
$$

A standard argument [7 implies $A: K \rightarrow C[0,1]$ is continuous and completely continuous. Next we show $A: K \rightarrow K$. If $u \in K$, then clearly $A u(t) \geq 0$ for $t \in[0,1]$. Also notice that

$$
\left\{\begin{array}{l}
(A u)^{(n)}(t) \leq 0 \quad \text { on }(0,1) \\
A u(0)=\frac{1}{m} \\
(A u)^{(i)}(0)=0, \quad 1 \leq i \leq n-2, \\
(A u)^{(p)}(1)=0
\end{array}\right.
$$


and so Theorem 1.1 implies $A u(t) \geq t^{n-1}|A u|_{0}$ for $t \in[0,1]$. Consequently $A u \in K$ so $A: K \rightarrow K$. Let

$$
\Omega_{1}=\left\{u \in C[0,1]:|u|_{0}<r\right\} \text { and } \Omega_{2}=\left\{u \in C[0,1]:|u|_{0}<R\right\} .
$$

We first show

$$
|A y|_{0} \leq|y|_{0} \text { for } y \in K \cap \partial \Omega_{1} .
$$

To see this let $y \in K \cap \partial \Omega_{1}$ so $y \in K,|y|_{0}=r$ and $y(t) \geq t^{n-1} r$ for $t \in[0,1]$. Also notice

$$
g^{\star}(y(t))+h(y(t)) \leq g(y(t))+h(y(t)) \text { for } t \in(0,1)
$$

since $g$ is nonincreasing on $(0, \infty)$. Now for $t \in[0,1]$,

$$
\begin{aligned}
A y(t) & =\frac{1}{m}+\int_{0}^{1} k(t, s) \phi(s)\left[g^{\star}(y(s))+h(y(s))\right] d s \\
& \leq \epsilon+\int_{0}^{1} k(t, s) \phi(s)[g(y(s))+h(y(s))] d s \\
& \leq \epsilon+\left\{1+\frac{h(r)}{g(r)}\right\} \int_{0}^{1} k(t, s) \phi(s) g(y(s)) d s \\
& \leq \epsilon+\left\{1+\frac{h(r)}{g(r)}\right\} K_{0} g(r) \int_{0}^{1} k(t, s) \phi(s) g\left(s^{n-1}\right) d s \\
& \leq \epsilon+[g(r)+h(r)] K_{0} \sup _{t \in[0,1]} \int_{0}^{1} k(t, s) \phi(s) g\left(s^{n-1}\right) d s
\end{aligned}
$$

using (2.3), (2.4), (2.6) and the fact that $y(t) \geq t^{n-1} r$ for $t \in[0,1]$. Consequently

$$
|A y|_{0} \leq \epsilon+[g(r)+h(r)] K_{0} c_{0}<r=|y|_{0}
$$

using (2.11). Hence (2.16) is true.

Next we show

$$
|A y|_{0} \geq|y|_{0} \text { for } y \in K \cap \partial \Omega_{2} .
$$

To see this let $y \in K \cap \partial \Omega_{2}$ so $|y|_{0}=R$ and $y(t) \geq t^{n-1} R$ for $t \in[0,1]$. In particular

$$
y(t) \in\left[\frac{R}{4^{n-1}}, R\right] \text { for } t \in\left[\frac{1}{4}, 1\right] .
$$

Also for $s \in\left[\frac{1}{4}, 1\right]$ we have

$$
g^{\star}(y(s))+h(y(s))=g(y(s))+h(y(s))
$$


since $y(s) \geq \frac{R}{4^{n-1}}>\frac{1}{m}$ for $s \in\left[\frac{1}{4}, 1\right]$. With $\sigma$ as defined in (2.10) we have, using (2.18) and (2.9),

$$
\begin{aligned}
A y(\sigma) & =\frac{1}{m}+\int_{0}^{1} k(\sigma, s) \phi(s)\left[g^{\star}(y(s))+h(y(s))\right] d s \\
& \geq \int_{\frac{1}{4}}^{1} k(\sigma, s) \phi(s)\left[g^{\star}(y(s))+h(y(s))\right] d s \\
& =\int_{\frac{1}{4}}^{1} k(\sigma, s) \phi(s)[g(y(s))+h(y(s))] d s \\
& =\int_{\frac{1}{4}}^{1} k(\sigma, s) \phi(s)\left\{1+\frac{h(y(s))}{g(y(s))}\right\} g(y(s)) d s \\
& \geq\left\{1+\frac{h\left(\frac{R}{4^{n-1}}\right)}{g\left(\frac{R}{4^{n-1}}\right)}\right\} g(R) \int_{\frac{1}{4}}^{1} k(\sigma, s) \phi(s) d s \\
& \geq R=|y|_{0}
\end{aligned}
$$

and so $|A y|_{0} \geq|y|_{0}$. Hence (2.17) is true.

Now Theorem 1.5 implies $A$ has a fixed point $y_{m} \in K \cap\left(\overline{\Omega_{2}} \backslash \Omega_{1}\right)$, i.e. $r \leq$ $\left|y_{m}\right|_{0} \leq R$. In fact $\left|y_{m}\right|_{0}>r$ (this is a standard argument since (2.11) holds [see the end of this Theorem]) and $y_{m}(t) \geq \frac{1}{m}$ for $t \in[0,1]$ (see (2.15)). Consequently $(2.13)^{m}$ (and also $(2.12)^{m}$ ) has a solution $y_{m} \in C^{n-1}[0,1] \cap C^{n}(0,1), y_{m} \in K$, with

$$
\frac{1}{m} \leq y_{m}(t) \text { for } t \in[0,1], r<\left|y_{m}\right|_{0} \leq R, \text { and } y_{m}(t) \geq t^{n-1} r \text { for } t \in[0,1]
$$

Also notice for $t \in(0,1)$ that

$$
-y_{m}^{(n)}(t)=\phi(t)\left[g\left(y_{m}(t)\right)+h\left(y_{m}(t)\right)\right] \leq \phi(t) g\left(t^{n-1} r\right)\left\{1+\frac{h(R)}{g(R)}\right\} .
$$

It is immediate from (2.19) and (2.20) (with of course (2.5)) that

$$
\left\{y_{m}\right\}_{m \in N_{0}} \text { is a bounded, equicontinuous family on }[0,1] \text {. }
$$

The Arzela-Ascoli Theorem guarantees the existence of a subsequence $N$ of $N_{0}$ and a function $y \in C[0,1]$ with $y_{m}$ converging uniformly on $[0,1]$ to $y$ as $m \rightarrow \infty$ through $N$. Also $y(0)=0, r \leq|y|_{0} \leq R$ and $y(t) \geq t^{n-1} r$ for $t \in[0,1]$. In particular $y>0$ on $(0,1]$. Now $y_{m}, m \in N$, satisfies

$$
y_{m}(t)=\frac{1}{m}+\int_{0}^{1} k(t, s) \phi(s)\left[g\left(y_{m}(s)\right)+h\left(y_{m}(s)\right)\right] d s \text { for } t \in[0,1] .
$$

Also as in (2.20),

$$
\phi(s)\left[g\left(y_{m}(s)\right)+h\left(y_{m}(s)\right)\right] \leq \phi(s) g\left(s^{n-1} r\right)\left\{1+\frac{h(R)}{g(R)}\right\} \in L^{1}[0,1] .
$$


Now let $m \rightarrow \infty$ through $N$ in (2.22) to obtain (here we use the Lebesgue Dominated Convergence Theorem),

$$
y(t)=\int_{0}^{1} k(t, s) \phi(s)[g(y(s))+h(y(s))] d s \text { for } t \in[0,1] .
$$

From (2.23) we deduce immediately that $y \in C^{n-1}[0,1] \cap C^{n}(0,1), y^{(n)}(t)+$ $\phi(t)[g(y(t))+h(y(t))]=0$ for $0<t<1, y^{(i)}(0)=0$ for $1 \leq i \leq n-2$ and $y^{(p)}(1)=0$. Finally $|y|_{0}>r$. To see this suppose $|y|_{0}=r$. Then for $t \in[0,1]$,

$$
\begin{aligned}
y(t) & =\int_{0}^{1} k(t, s) \phi(s)[g(y(s))+h(y(s))] d s \\
& \leq\left\{1+\frac{h(r)}{g(r)}\right\} \int_{0}^{1} k(t, s) \phi(s) g(y(s)) d s \\
& \leq\left\{1+\frac{h(r)}{g(r)}\right\} K_{0} g(r) \sup _{t \in[0,1]} \int_{0}^{1} k(t, s) \phi(s) g\left(s^{n-1}\right) d s
\end{aligned}
$$

since $y(t) \geq t^{n-1} r$ for $t \in[0,1]$. Thus

$$
r=|y|_{0} \leq[g(r)+h(r)] K_{0} c_{0},
$$

and this contradicts (2.7).

Remark 2.3. If in (2.9) we have $R<r$, then (2.1) has a solution $y \in C^{n-1}[0,1] \cap$ $C^{n}(0,1)$ with $y>0$ on $(0,1]$ and $R \leq|y|_{0}<r$. The argument is similar to that in Theorem 2.2 except here we use the other half of Theorem 1.5.

Remark 2.4. It is also possible to use the ideas in Theorem 2.2 to discuss the more general problem

$$
\left\{\begin{array}{l}
y^{(n)}(t)+\phi(t) f(t, y(t))=0,0<t<1 \\
y^{(i)}(0)=0, \quad 0 \leq i \leq n-2 \\
y^{(p)}(1)=0
\end{array}\right.
$$

Theorem 2.3. Assume (2.2)-(2.7) and (2.9) hold. Then (2.1) has two solutions $y_{1}, y_{2} \in C^{n-1}[0,1] \cap C^{n}(0,1)$ with $y_{1}>0, y_{2}>0$ on $(0,1]$ and $\left|y_{1}\right|_{0}<r<$ $\left|y_{2}\right|_{0} \leq R$.

Proof. The existence of $y_{1}$ follows from Theorem 2.1 and the existence of $y_{2}$ follows from Theorem 2.2.

Example 2.1. The singular boundary value problem

$$
\left\{\begin{array}{l}
y^{\prime \prime}+\frac{1}{3}\left(y^{-\alpha}+y^{\beta}+1\right)=0 \text { on }(0,1) \\
y(0)=y^{\prime}(1)=0,0<\alpha<1<\beta
\end{array}\right.
$$

has two solutions $y_{1}, y_{2} \in C^{1}[0,1] \cap C^{2}(0,1)$ with $y_{1}>0, y_{2}>0$ on $(0,1]$ and $\left|y_{1}\right|_{0}<1<\left|y_{2}\right|_{0}$.

To see this we will apply Theorem 2.3 with $n=2, p=1, \phi=\frac{1}{3}, g(u)=u^{-\alpha}$ and $h(u)=u^{\beta}+1$. Clearly (2.2), (2.3), (2.4), (2.5) (since $0<\alpha<1$ ) and (2.6) (with $K_{0}=1$ ) hold. In this situation

$$
k(t, s)= \begin{cases}s, & 0 \leq s \leq t \\ t, & t \leq s \leq 1\end{cases}
$$


So

$$
c_{0}=\frac{1}{3} \sup _{t \in[0,1]}\left[\int_{0}^{t} s^{1-\alpha} d s+t \int_{t}^{1} s^{-\alpha} d s\right]=\frac{1}{3} \frac{1}{2-\alpha} .
$$

Now (2.7) holds (with $r=1$ ) since if $r=1$,

$$
\frac{r}{g(r)+h(r)}=\frac{r}{r^{-\alpha}+r^{\beta}+1}=\frac{1}{3}>\frac{1}{3} \frac{1}{2-\alpha} .
$$

Finally since (note $\beta>1$ ),

$$
\lim _{R \rightarrow \infty} \frac{R g\left(\frac{R}{4}\right)}{g(R) g\left(\frac{R}{4}\right)+g(R) h\left(\frac{R}{4}\right)}=\lim _{R \rightarrow \infty}\left(\frac{R^{\alpha+1} 4^{\alpha}}{4^{\alpha}+4^{-\beta} R^{\alpha+\beta}+R^{\alpha}}\right)=0,
$$

there exists $R>1$ so that (2.9) holds. The result now follows from Theorem 2.3.

Remark 2.5. The result in Theorem 2.2 extends to the nonsingular problem, i.e. when $g \equiv 0$. The proof is a lot easier in this case. In fact one can deal directly here with $(2.1)$ (with $g \equiv 0$ ), i.e. there is no need to modify the problem as in $(2.12)^{m}$ (with $g \equiv 0$ ).

Next we discuss the singular $(p, n-p)$ focal boundary value problem

$$
\left\{\begin{array}{l}
(-1)^{n-p} y^{(n)}(t)=\phi(t)[g(y(t))+h(y(t))], \quad 0<t<1 \\
y^{(i)}(0)=0, \quad 0 \leq i \leq p-1 \\
y^{(i)}(1)=0, \quad p \leq i \leq n-1
\end{array}\right.
$$

Theorem 2.4. Assume (2.2)-(2.4) and (2.6) are satisfied. In addition assume

$$
\int_{0}^{1} \phi(s) g\left(s^{p}\right) d s<\infty
$$

and

$$
\text { there exists a constant } r>0 \text { with } \frac{r}{g(r)+h(r)}>b_{0} K_{0}
$$

hold; here

$$
b_{0}=\sup _{t \in[0,1]} \int_{0}^{1}(-1)^{n-p} G(t, s) \phi(s) g\left(s^{p}\right) d s
$$

where $G(t, s)$ is the Green's function for (1.5). Then (2.25) has a solution $y \in$ $C^{n-1}[0,1] \cap C^{n}(0,1)$ with $y>0$ on $(0,1]$ and $|y|_{0}<r$.

Proof. The result follows from Theorem 1.3.

Theorem 2.5. Assume (2.2)-(2.4), (2.6), (2.26) and (2.27) hold. In addition suppose

there exists $R>r$ with $\frac{R g\left(\frac{R}{4^{p}}\right)}{g(R) g\left(\frac{R}{4^{p}}\right)+g(R) h\left(\frac{R}{4^{p}}\right)} \leq \int_{\frac{1}{4}}^{1}(-1)^{n-p} G(\sigma, s) \phi(s) d s$; here $0 \leq \sigma \leq 1$ is such that

$$
\int_{\frac{1}{4}}^{1}(-1)^{n-p} G(\sigma, s) \phi(s) d s=\sup _{t \in[0,1]} \int_{\frac{1}{4}}^{1}(-1)^{n-p} G(t, s) \phi(s) d s .
$$

Then (2.25) has a solution $y \in C^{n-1}[0,1] \cap C^{n}(0,1)$ with $y>0$ on $(0,1]$ and $r<|y|_{0} \leq R$. 
Proof. The proof is similar to that in Theorem 2.2. In this case $K=\left\{u \in C[0,1]: u(t) \geq 0\right.$ for $t \in[0,1]$ and $u(t) \geq t^{p}|u|_{0}$ for $\left.t \in[0,1]\right\}$.

Theorem 2.6. Assume (2.2)-(2.4), (2.6), (2.26), (2.27) and (2.29) hold. Then (2.25) has two solutions $y_{1}, y_{2} \in C^{n-1}[0,1] \cap C^{n}(0,1)$ with $y_{1}>0, y_{2}>0$ on $(0,1]$ and $\left|y_{1}\right|_{0}<r<\left|y_{2}\right|_{0} \leq R$.

Remark 2.6. By imposing other conditions on the nonlinearity $h+g$ it is easy to establish the existence of more than two solutions to (2.1) and (2.25).

\section{REFERENCES}

[1] R. P. Agarwal and D. O'Regan, Singular boundary value problems for superlinear second order ordinary and delay differential equations, Jour. Differential Eqns., 130(1996), 333355. MR 97g:34028

[2] R. P. Agarwal and D. O'Regan, Right focal singular boundary value problems, ZAMM 79(1999), 363-373. CMP 99:14

[3] R. P. Agarwal, D. O'Regan and V. Lahshmikantham, Singular $(p, n-p)$ focal and $(n, p)$ higher order boundary value problems, Nonlinear Analysis, to appear.

[4] R. P. Agarwal, D. O'Regan and P. J. Y. Wong, Positive solutions of Differential, Difference and Integral equations, Kluwer, Dordrecht, 1999. CMP 99:10

[5] P. W. Eloe and J. Henderson, Singular nonlinear boundary value problems for higher order ordinary differential equations, Nonlinear Analysis, 17(1991), 1-10. MR 93b:34034

[6] L. H. Erbe, S. Hu and H. Wang, Multiple positive solutions of some boundary value problems, Jour. Math. Anal. Appl., 184(1994), 640-648. MR 95f:34023

[7] D. O'Regan, Existence Theory for Nonlinear Ordinary Differential Equations, Kluwer, Dordrecht, 1997. MR 98h:34042

Department of Mathematics, National University of Singapore, 10 Kent Ridge CresCENT, Singapore 119260

E-mail address: matravip@nus.edu.sg

Department of Mathematics, National University of Ireland, Galway, Ireland

E-mail address: donal.oregan@nuigalway.ie 